\title{
Monitoring of Blood Glucose Level in Surgical Intensive Care Unit Patients with Sepsis
}

\author{
ABDULLAH N. EL-ORABY, M.Sc.; SOHAIR M. SOLIMAN, M.D.; AHMED A. EL-DABA, M.D. and \\ WAIL E. MESBAH, M.D. \\ The Department ofAnesthesiology, Faculty of Medicine, Tanta University
}

\begin{abstract}
Background: Sepsis now is defined as evidence of infection plus life-threatening organ dysfunction, clinically characterized by an acute changes of 2 points or greater in the SOFA score. The new clinical criteria for septic shock include sepsis with fluid-unresponsive hypotension, serum lactate level greater than $2 \mathrm{mmol} / \mathrm{L}$, and the need of vasopressor to maintain mean arterial pressure of $65 \mathrm{mmHg}$ or greater. One of the most important metabolic changes in sepsis is hyperglycemia arising from muscle glycolysis and lipolysis, and subsequent gluconeogenesis and glycolysis in the liver. The other feature of metabolic changes in sepsis is hyperlactatemia due to glycolysis in muscle caused by counter regulatory hormones and cytokines. Gcontrol to a moderately tight range (140-200mg/dl) is not inferior to euglycemia and clearly safer in critically ill patients.
\end{abstract}

Aim of Study: The aim of this study is monitoring of blood glucose level in Surgical Intensive Care Unit patients with sepsis.

Patients and Methods: This study was carried out in Tanta University Hospitals at Surgical Intensive Care Unit (SICU) for six months (from October 2017 to March 2018) after a written informed consent was obtained from the patients relatives, 100 patients of both sex admitted to SICU suffering from sepsis were enrolled in the study. Everyone had a secret code number.

Results: Our results showed that no significant correlation between C-Peptide level \& RBS in hyperglycemic patients, -ve significant correlation between C-Peptide level \& RBS in hypoglycemic patients, significant decrease in MAP of hypoglycemic cases more than hyperglycemic cases, significant increase in HR of hyperglycemic cases more than hypoglycemic, significant increase in mortality in hypoglycemic patients as compared to hyperglycemic patients.

Conclusion: We concluded that frequent blood glucose measurement is one element of the routine intensive monitoring that all critically ill patients receive following admission to intensive care units. Transient increase in blood glucose concentration (hyperglycemia) is very common in this patient. Although decrease in blood glucose concentration (hypogly-

Correspondence to: Dr. Abdullah N. El-Oraby, The Department of Anesthesiology, Faculty of Medicine, Tanta University cemia) is rare but has very bad prognosis with significant increase in mortality rate.

Key Words: Sepsis - Hyperglycemia - Blood Glucose.

\section{Introduction}

SEPSIS is a life-threatening condition that arises when the body's response to infection injures its own tissues and cause organs dysfunction [1].

There are many pathophysiological changes during sepsis and septic shock, and one of the most striking is metabolic derangement. Among the metabolic changes, hyperglycemia is the most important $[2,3]$

Hyperglycemia is a common feature of the critically ill in general and of patients with sepsis in particular. Even a moderate degree of hyperglycemia appears harmful for the outcome of critically ill patients, since maintenance of normoglycemia (blood glucose levels $<$ or $=110 \mathrm{mg} / \mathrm{dL}$ ) with intensive insulin therapy has shown to improve survival and reduce morbidity in prolonged critically ill patients in Surgical Intensive Care Units [2]

But some cases of sepsis may be complicated by the occurrence of hypoglycemia, which is caused by dysbalance between glucose production and glucose consumption $[4,5]$.

The pathophysiological explanation for this dysregulation may be related to classical endocrinal mechanisms, like hyperinsulinemia [6]

\section{Patients and Methods}

This study was carried out in Tanta University Hospitals at Surgical Intensive Care Unit (SICU) for six months after a written informed consent was obtained from the patients relatives, 100 
patients of both sex admitted to SICU suffering from sepsis were enrolled in the study. Everyone had a secret code number.

The following measurements were recorded at admission after inclusion in our study:

- Blood culture, CBC, CRP.

- Lactic acid level.

- Liver function $\&$ kidney function tests.

The following routine examinations \& investigations were recorded for 24 hours after inclusion in our study:

- Mean Arterial blood Pressure (MAP) every 2 hours.

- Heart rate (b/min) every 2 hours.

- Arterial blood glucose level every 2 hours.

- Arterial C-peptide and insulin level once hyperglycemia or hypoglycemia occur by ELIZA.

\section{Fluid management:}

- Administer 30ml/Kg Ringer's solution within the first 3 hours targeting MAP $>65 \mathrm{mmHg}$.

- If MAP elevate $>65 \mathrm{mmHg}$, give maintenance dose of Ringer's solution $(2 \mathrm{ml} / \mathrm{kg} / \mathrm{hr})$.

- If MAP still $<65 \mathrm{mmHg}$, start noradrenaline infusion at $0.01 \mu \mathrm{g} / \mathrm{kg} / \mathrm{min}$ and titrate up to $0.1-0.2 \mu \mathrm{g} /$ $\mathrm{kg} / \mathrm{min}(1)$.

Glucose control:

- The blood glucose level in ICU patients with sepsis should be maintained between 140 and $180 \mathrm{mg} / \mathrm{dl}$.

- Serum glucose will be monitored every 2 hours.

- If hyperglycemia occure $(>180 \mathrm{mg} / \mathrm{dl})$, administer insulin infusion targeting blood glucose level (140-180mg/dl).

- If hypoglycemia occure, administer $100 \mathrm{ml}$ glucose $25 \%$.

\section{Results}

Glucose level of 100 patients:

There were fifty three patients (53\%) experienced hyperglycemia. While, eight patients $(8 \%)$ experienced hypoglycemia. But, thirty nine patients (39\%) experienced normoglycemia.

Table (1), Fig. (2): Blood Cultures of 100 patients: There were 39 culture-negative patients $(39 \%) \& 61$ culture-positive patients $(61 \%)$.

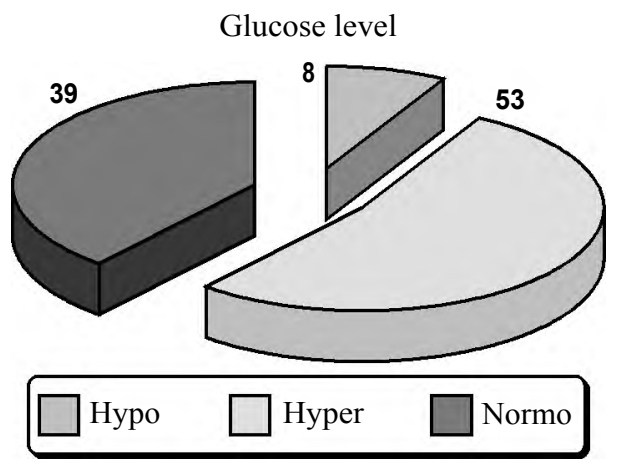

Fig. (1): Glucose level.

Table (1): Blood cultures.

\begin{tabular}{lll}
\hline Blood cultures & $\mathrm{N}$ & $\%$ \\
\hline Culture negative & 39 & 39.0 \\
Proteus & 2 & 2.0 \\
E. Coli & 11 & 11.0 \\
Enterococcus & 3 & 3.0 \\
Klebsiella pneumoniae & 13 & 13.0 \\
klebsiella \& proteus & 7 & 7.0 \\
Pseudomonas aeruginosa & 6 & 6.0 \\
Staph. aureus & 12 & 12.0 \\
Strept. pneumonia & 7 & 7.0 \\
\hline Total & 100 & 100.0 \\
\hline
\end{tabular}
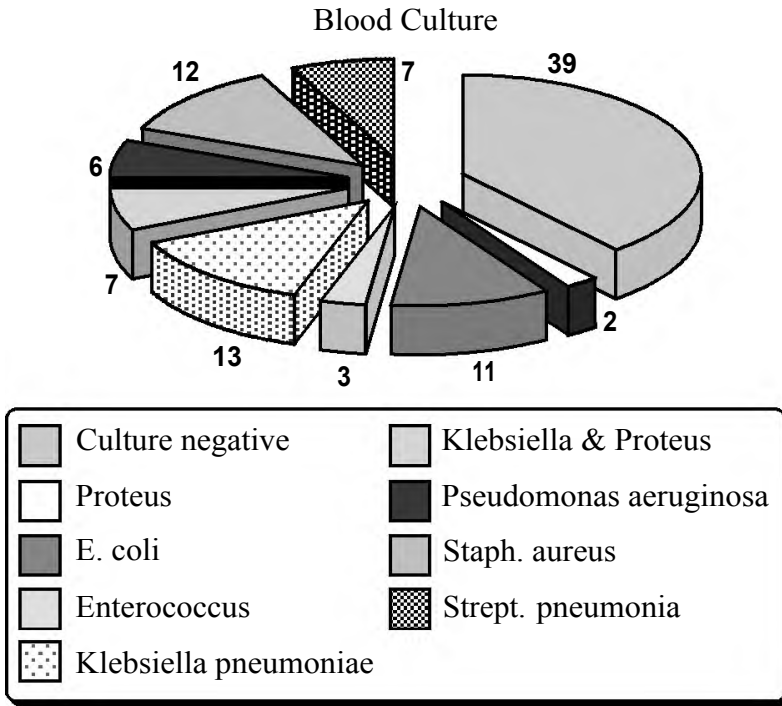

Fig. (2): Blood culture.

Blood samples of two patients (2\%) show proteus, eleven patients $(11 \%)$ show E.Coli, three patients (3\%) show Enterococci, thirteen patients (13\%) show Klebsiella pneumoniae, seven patients (7\%) show Klebsiella \& Proteus, six patients $(6 \%)$ show Pseudomonas aeruginosa, twelve patients (12\%) show Staph. Aureus. finally, seven patients (7\%) show Strept. Pneumonia.

Fig. (3): Correlation between C-Peptide level $\&$ RBS in hyperglycemic patients: The base line (at admission), random blood sugar ranged between 
$190-433 \mathrm{mg} / \mathrm{dl}$ with a mean value $261.10 \pm 52.10$ $\mathrm{mg} / \mathrm{dl}$. While C-Peptide ranged between 1.2-4.10 $\mathrm{ng} / \mathrm{ml}$ with a mean value $2.41 \pm .82 \mathrm{ng} / \mathrm{ml}$. There was no significant correlation between C-Peptide level \& RBS in hyperglycemic patients (normal C-Peptide level with hyperglycemia).

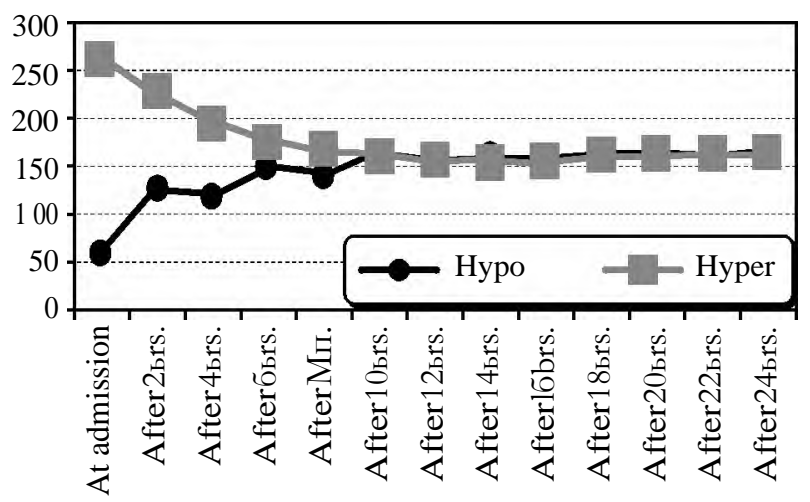

Fig. (3): Comparison between measurements of RBS every $2 \mathrm{hrs}$ after admission for $24 \mathrm{hrs}$ in hypoglycemic \& hyperglycemic patients.

Table (2), Fig. (4): Correlation between CPeptide level \& RBS in hypoglycemic patients: The base line (at admission), random blood sugar ranged between $35-72 \mathrm{mg} / \mathrm{dl}$ with a mean value $57.00 \pm 12.98 \mathrm{mg} / \mathrm{dl}$. While C-Peptide ranged between $9.2-26.3 \mathrm{ng} / \mathrm{ml}$ with a mean value $19.3 \pm 6$ $\mathrm{ng} / \mathrm{ml}$. There was -ve significant correlation between C-Peptide level \& RBS in hypoglycemic patients.

Three patients (37.5\%) of hypoglycemic patients were died in 1 st $24 \mathrm{hrs}$. While, hyperglycemic patients show no mortality $(0 \%)$. There was statistically significant increase in mortality in hypoglycemic patients as compared to hyperglycemic patients.

Table (2): Comparison between hypoglycemic \& hyperglycemic patients as regard mortality in 1 st $24 \mathrm{hrs}$ after admission

\begin{tabular}{clll}
\hline Mortality & Hypo & Hyper & Total \\
\hline Alive: & & & \\
$\mathrm{N}$ & 5 & 53 & 58 \\
$\%$ & $62.5 \%$ & $100.0 \%$ & $95.1 \%$ \\
Died: & & & \\
$\mathrm{N}$ & 3 & 0 & 3 \\
$\%$ & $37.5 \%$ & $.0 \%$ & $4.9 \%$ \\
Total: & & & \\
$\mathrm{N}$ & 8 & 53 & 61 \\
$\%$ & $100.0 \%$ & $100.0 \%$ & $100.0 \%$ \\
Chi-square: & & & \\
$\chi^{2}$ & & 20.921 & \\
$p$-value & & $0.001^{*}$ & \\
\hline
\end{tabular}

*: Significant $p$-value $<0.05$.
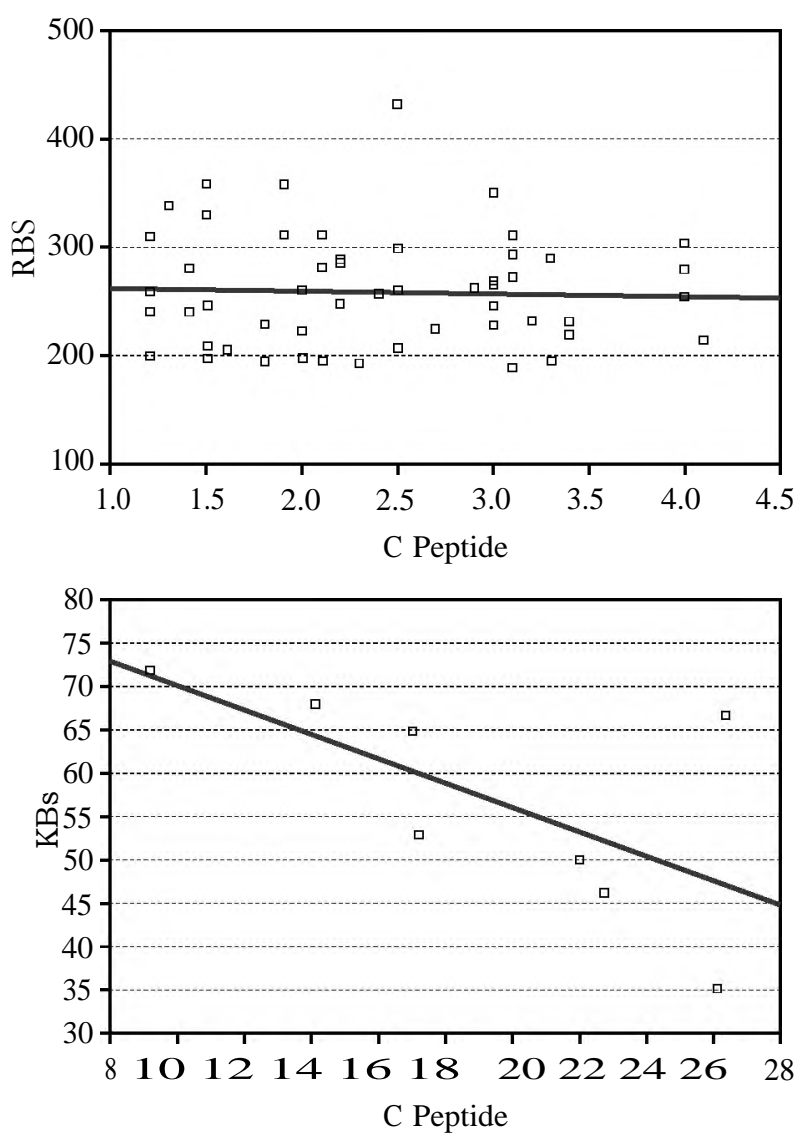

Fig. (4): Comparison between C-Peptide level in hypoglycemic $\&$ hyperglycemic patients.

\section{Discussion}

Sepsis is the $10^{\text {th }}$ leading cause of death and one of the leading causes for admission to the Intensive Care Unit. Sepsis and Septic Shock are common problems encountered in the ICU. In fact, septic shock with Multiorgan Dysfunction (MODS) is the leading cause of death in non-coronary ICU's [7].

Hyperglycemia is common in critically ill patients, eventhose patients who have not been previously diagnosed with diabetes. Clinicians have increasingly appreciated the impact of hyperglycemia in patients with diabetes, as well as Stressinduced Hyperglycemia (SIH) or hospital-related hyperglycemia [8]. Hypoglycemia is rare with sepsis but has very bad prognosis [9] .

In this study, we monitor blood glucose level in surgical intensive care unit patients with sepsis, observe vitality, manage glucose level abnormality $\&$ correlate between C-Peptide, insulin level and glucose level.

Our results were in agreement with Tiruvoipati et al.; [10] who studied Increased blood glucose in 
patients with sepsis. The study population comprised 297 septic patients admitted to the Intensive Care Unit of an Australian Teaching Hospital over a five year period. All patients had blood glucose concentration estimated at least once every six hours for the duration of their stay in intensive care. A blood gas analyzer sited in the unit was used for these measurements. Stress hyperglycemia was diagnosed if the mean of these blood glucose estimations exceeded $6.9 \mathrm{mmol} / \mathrm{L}$. By this criterion 204 of the 297 (68.7\%) patients had stress hyperglycaemia and $93(31.3 \%)$ did not.

Our results were in agreement with de Prost et al.; [11] who studied unrevealing culture-negative severe sepsis. In this issue of Critical Care, Phua and Colleagues reported on a large prospective cohort study of patients $(n=1,001)$ presenting with severe sepsis on ICU admission and compared the characteristics and outcomes of culture-negative versus culture-positive episodes. Their main findings were that culture-negative sepsis occurred in $41.5 \%$ of cases.

Our study was in agreement with Jones et al.; [12] who studied The clinical utility of C-peptide measurement in the care of patients with diabetes which reveal C-peptide is produced in equal amounts to insulin and is the best measure of endogenous insulin secretion in patients with diabetes. Measurement of insulin secretion using Cpeptide can be helpful in clinical practice: Differences in insulin secretion are fundamental to the different treatment requirements of Type 1 and Type 2 diabetes.

We claim that hyperglycemia is due to Gluconeogenesis duo to insulin resistance under the effect of counterregulatory hormones. But hypoglycemia duo to increase insulin secretion.

Romijn et al.; [13] who studied Hypoglycemia, hormones and cytokines in fatal meningococcal septicemia. A unique case report with sequential measurements of the plasma concentrations of glucoregulatory hormones, interleukin- 6 and tumor necrosis factor during development of hypoglycemia in fatal meningococcemia is presented. This study reveal that there was no defect in hypoglycemic counterregulatory hormones, judged by high concentrations of glucagon, catecholamines, growth hormone and cortisol. Hyperinsulinemia, which has been suggested to be the cause of septic hypoglycemia.

In contrast to our study van der Crabben et al.; [14] who studied Early Endotoxemia Increases Peripheral and Hepatic Insulin Sensitivity in Healthy
Humans. Glucose metabolism was measured during two hyperinsulinemic (insulin levels of $100 \mathrm{pmol} /$ liter (low-dose clamp) and 400pmol/liter (mediumdose clamp)) euglycemic ( $5 \mathrm{mmol} / \mathrm{liter}$ ) clamps on two occasions: Without or with Lipopolysaccharide (LPS). Volunteers $(n=18)$ were studied during euglycemia (5mmol/liter) during either a plasma insulin level of 100pmol/liter (low-dose hyperinsulinemic clamp) or an insulin level of 400pmol/ liter (medium-dose hyperinsulinemic clamp) to study hepatic and peripheral insulin sensitivity, respectively. In the control group, volunteers $(n=6)$ were studied twice, i.e. during both clamps. Because it is not feasible to administer LPS twice to the same subjects, six volunteers underwent the lowdose hyperinsulinemic clamp concomitant with LPS, and six other volunteers underwent the medium-dose hyperinsulinemic clamp aimed at an insulin level of 400pmol/liter plus LPS. This study show after administration of LPS, peripheral and hepatic insulin sensitivity increase. This may contribute to the hypoglycemia occurring in some patients with critical illness.

Krinsley et al.; [15] who studied Mild hypoglycemia is independently associated with increased mortality in the critically ill. Data from 6,240 patients was analyzed: 3,263 admitted to Stamford Hospital (ST), 2,063 admitted to three institutions in The Netherlands (NL) and 914 who participated in the GLUCONTROL trial (GL). The percentage of patients with hypoglycemia varied from $18 \%$ to $65 \%$ among the different cohorts. This study reveal that mild hypoglycemia was associated with a significantly increased risk of mortality in an international cohort of critically ill patients. Efforts to reduce the occurrence of hypoglycemia in critically ill patients may reduce mortality. The possible causes of this may be due to large swings in BG, as observed when hypoglycemia is aggressively treated with a large amount of intravenous glucose, may be associated with cellular damages. Unfortunately, data detailing the amount of glucose given to treat hypoglycemia and the neurological status of the patients in our three cohorts were not available. The detrimental effects of hypoglycemia are well documented in the brain. Indeed, glucose is the preferential energetic substrate in the brain. The absence of cerebral stores of glucose and the diffusive character of transport imply that the glucose concentration in neurons and glial cells is entirely determined by BG. Therefore, brain injured patients are at higher risk of hypoglycemia-related damage; conversely, hypoglycemia also induces brain dysfunction even in patients without prior cerebral compromise. 


\section{Conclusion:}

We concluded that frequent blood glucose measurement is one element of the routine intensive monitoring that all critically ill patients receive following admission to intensive care units. Transient increase in blood glucose concentration (hyperglycemia) is very common in this patient. Although decrease in blood glucose concentration (hypoglycemia) is rare but has very bad prognosis with significant increase in mortality rate.

\section{References}

1- MARTIN G.S.: Sepsis, severe sepsis and septic shock: Changes in incidence, pathogens and outcomes. Expert Review of Anti-Infective Therapy, 10 (6): 701-6, 2012.

2- PERMAN S.M., GOYAL M. and GAIESKI D.F.: Initial Emergency Department diagnosis and management of adult patients with severe sepsis and septic shock. Scandinavian Journal of Trauma, Resuscitation and Emergency Medicine, 20 (1): 41, 2012.

3- VANDIJCK D., DECRUYENAERE J. and BLOT S.: The value of sepsis definitions in daily ICU-practice. Acta Clinica Belgica, 61 (5): 220-6, 2006.

4- BOEDICKER J.Q., LI L., KLINE T.R. and ISMAGILOV R.F.: Detecting bacteria and determining their susceptibility to antibiotics by stochastic confinement in nanoliter droplets using plug-based microfluidics. Lab on a Chip., 8 (8): 1265-72, 2008.

5- ALBERTI C., BRUN-BUISSON C., BURCHARDI H., MARTIN C., GOODMAN S., ARTIGAS A., et al.: Epidemiology of sepsis and infection in ICU patients from an international multicentre cohort study. Intensive Care Medicine, 28 (2): 108-21, 2002.

6- DANIKAS D., KARAKANTZA M., THEODOROU G., SAKELLAROPOULOS G. and GOGOS C.: Prognostic value of phagocytic activity of neutrophils and monocytes in sepsis. Correlation to CD64 and CD14 antigen expres- sion. Clinical \& Experimental Immunology, 154 (1): 87 97, 2008.

7- ZHOU X., WU F., YE Y. and LI J.: Classifying reasons for mortality in septic patients by limited categories, still a long way to go. Journal of Critical Care, 44: 466-7, 2018.

8- KRINSLEY J., SCHULTZ M., SPRONK P., HARMSEN R., HOUCKGEEST F.V.B., VAN DER SLUIJS J., et al.: Mild hypoglycemia is independently associated with increased mortality in the critically ill. Critical Care, 15 (1): p. 397, 2011.

9- JAN I.S., TSAI T.H., CHEN J.M., JERNG J.S., HSU H.F., HUNG P.L., et al.: Hypoglycemia associated with bacteremic pneumococcal infections. International Journal of Infectious Diseases, 13 (5): 570-6, 2009.

10-TIRUVOIPATI R., GUPTA S., PILCHER D. and BAILEY M.: Hypercapnia and hypercapnic acidosis in sepsis: Harmful, beneficial or unclear? Crit. Care Resusc., Jun., 20 (2): 94-100, 2018.

11- De PROST N., RAZAZI K. and BRUN-BUISSON C. Unrevealing culture-negative severe sepsis. Critical Care, 17 (5): 1001, 2013.

12- JONES A. and HATTERSLEY A.: The clinical utility of C-peptide measurement in the care of patients with diabetes. Diabetic Medicine, 30 (7): 803-17, 2013.

13- ROMIJN J.A., GODFRIED M.H., WORTEL C. and SAUERWEIN H.P.: Hypoglycemia, hormones and cytokines in fatal meningococcal septicemia. J. Endocrinol. Invest. 1990 Oct., 13 (9): 743-7.

14- VAN DER CRABBEN S.N., BLUMER R.M., STEGENGA M.E., ACKERMANS M.T., ENDERT E., TANCK M.W., et al.: Early endotoxemia increases peripheral and hepatic insulin sensitivity in healthy humans. The Journal of Clinical Endocrinology \& Metabolism, 94 (2): 463-8, 2009.

15- KRINSLEY J.S. and GROVER A.: Severe hypoglycemia in critically ill patients: Risk factors and outcomes. Critical Care Medicine, 35 (10): 2262-7, 2007.

\section{رصد مستوى السكرفى الدم فى مرضى العناية المركزة الجراحية

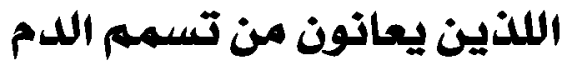

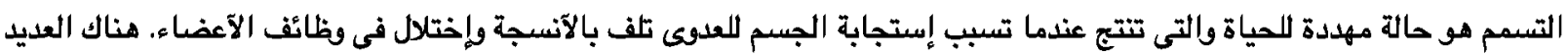

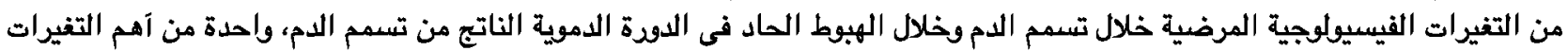

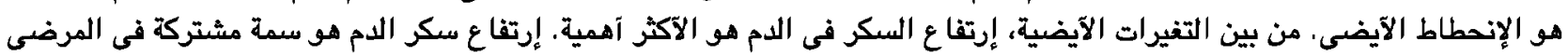

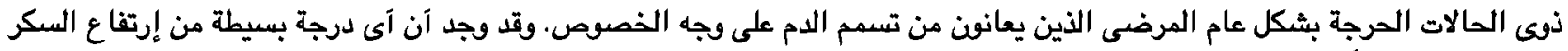

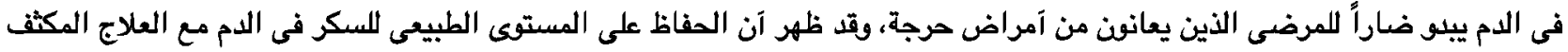

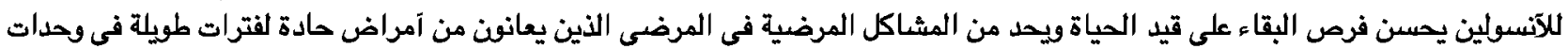

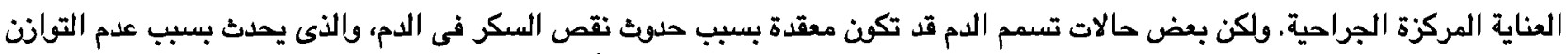

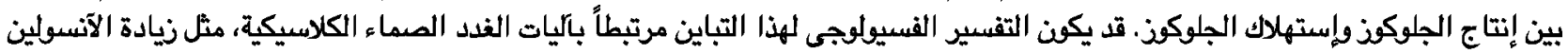

